\section{Aktuelle und zukünftige Funktechnologie}

\section{Zur Exposition der Bevölkerung mit elektromagnetischen Feldern}

\author{
von Gerd Friedrich, Forschungs- \\ gemeinschaft Funk, Bonn
}

Wir sind auf dem Weg in eine neue Mediengesellschaft. Überall sollen „,drahtlos“ Informationen, Waren- und Personen-Identifizierungen sowie Unterhaltungsangebote verfügbar sein. Folgt man den Ankündigungen von Medien-Experten in den einschlägigen Magazinen und Journalen, steht uns die eigentliche Explosion der Anwendungsvielfalt im Funkbereich erst noch bevor. „Schöne, neue Welten" für die persönliche aber auch öffentliche Kommunikation per Funk werden angekündigt. Diese ,totale" drahtlose Vernetzung trifft aber nicht nur auf Befürworter. Im Gegenteil, ,Funk"-Anwendungen sind in die Kritik geraten; sie rufen sogar Ängste über „Umweltverschmutzung“ und Gefährdung der Gesundheit hervor. Aber ist das gerechtfertigt? Die Frage nach eventuellen Beeinflussungen oder gar Schädigungen biologischer Systeme durch elektromagnetische Felder wird weltweit intensiv diskutiert und seit dem Start der zweiten Generation der Mobilfunktelefonie mit erheblichem finanziellen Aufwand ,beforscht“. Die Erkenntnisse sind in Rechtsverordnungen, Normen und Standards zum Schutz der Bevölkerung eingeflossen. Zusätzlich wurden Messungen zur Ermittlung der tatsächlichen „Befeldung“ (Exposition) der Öffentlichkeit durchgeführt und in Datenbanken erfasst. Dennoch stoppt die Diskussion in der Öffentlichkeit nicht. Populär wurde als Schlagwort für diese Debatte das Kunstwort „Elektrosmog".

\section{Mobile Kommunikation: Rückblick und Ausblick}

Kein Tag vergeht ohne Anpreisung von neuen Geräten und sensationellen Applikationen. Natürlich wird ,alles“ schneller, breitbandiger und billiger, damit wir auch ,alles“ ungeniert nutzen können. Wir werden vernetzt und dies fast nur noch drahtlos, sind permanent verfügbar, sind in der Lage, große Datenmengen zu empfangen und weiterzuverarbeiten, können interaktiv spielen und uns amüsieren mit dem, was Hollywood und andere Traumfabriken uns rund um die Uhr anbieten. Es soll keiner leer ausgehen. Die ,elektromagnetische Welle“, der Funk, macht dies alles möglich.

Für nahezu alle Einwohner Deutschlands ist das Mobiltelefon, liebevoll im Volksmund „Handy“ genannt, heute ein nicht mehr wegzudenkendes Kommunikationsmittel, das sogar beginnt, das Festnetz mehr und mehr zu substituieren. Die Anzahl der benutzten „Handys“ übersteigt inzwischen bei weitem die Zahl der Bevölkerung. Es ist kaum zu glauben, wie im Laufe der Zeit immer größere Datenmengen über alt bekannte Übertragungswege ,gejagt" werden können. Selbst „schmalbandige“ Funkkanäle werden raffiniertere Modulationen, Digitalisierung bzw. „Verwürfelungen“ aufgepeppt, um immer größere Datenmengen transportieren zu können.

Der Einsatz von Funkdiensten kam anfangs sehr schleichend, ja man kann behaupten, fast verschämt. Die Anfänge des öffentlichen Mobilfunks gehen auf das Jahr 1918 zurück, als im Auftrag der Deutschen Reichspost erste Sprechfunkversuche von fahrenden Zügen aus durchgeführt wurden („Eisenbahn-Telefonie“). Nach dem Krieg betrieb die Deutsche Bundespost zunächst verschiedene Versuchsnetze in Großstädten, an wichtigen Häfen, Autobahnen und Binnenwasserstraßen in den Frequenzbereichen $30 \mathrm{MHz}, 80 \mathrm{MHz}$ und $160 \mathrm{MHz} .1958$ war das Geburtsjahr des A-Netzes, als man sich entschied, ein öffentliches Mobilfunksystem in einheitlicher 160-MHz-Technik aufzubauen. Dies war ein Frequenz-Zugriffsverfahren (FDD $=$ Frequency Division Duplex) und hatte einen Duplex-Abstand von 4,5 MHz, um an dieser Stelle nur die wichtigsten funktechnischen Parameter anzuführen.

Über die analogen A-, B-, und C-Netze (450 MHz) der ersten Generation, die digitalen GSM-Netze der zweiten Generation (GSM Global System for Mobile Communications, $900 \mathrm{MHz}, 1800 \mathrm{MHz}$ and $1900 \mathrm{MHz}$ ) und deren Evolutionsschritte, die UMTS-Netze der dritten Generation mit ihren Weiterentwicklungen bis hin zu den Aktivitäten zur Konzipierung einer zukünftigen Technologie der vierten Generation: Die Entwicklungszyklen der Techniken folgen immer rascher. 
Die Weiterentwicklung des Mobilfunks ist durch das Ziel geprägt, höhere Datenraten bei gesteigerter Frequenzökonomie und günstigeren Kosten $\mathrm{zu}$ ermöglichen und damit neben den klassischen Sprachdiensten weitere Anwendungen im Internet- und Unterhaltungsbereich zu ermöglichen. Beispiele dafür sind der Zugriff auf bzw. die Versendung von Multimedia-Inhalten (z. B. Fotos, Filmschnipsel, Musik und Handy-TV).

Mit UMTS (Universal Mobile Telecommunications System), dem Mobilfunkstandard der dritten Generation (3G), wurden bereits wesentlich höhere Datenübertragungsraten erreicht als mit dem GSM-Mobilfunk der zweiten Generation (2G) und seinen Erweiterungen GPRS (General Packet Radio Services) und EDGE (Enhanced Data Rates for GSM Evolution) zur Beschleunigung der Datenübertragung. Aber auch die UMTS-Übertragungsverfahren konnten im Zuge der weiteren technischen Entwicklung und Standardisierung noch verbessert und beschleunigt werden; dazu gehören High Speed Downlink Packet Access (HSDPA) und High Speed Uplink Packet Access (HSUPA).

Parallel erfolgt die Entwicklung weiterer breitbandiger Funkzugänge zum Internet. Neben WLAN (Wireless Local Area Network) Hotspots mit zunehmend höheren Datenraten wird die Entwicklung von WiMAX (Worldwide Interoperability for Microwave Access) vorangetrieben, dessen Standards sowohl ortsfeste als auch mobile Systeme unterstützen sollen.

Um die Kommunikationsbedürfnisse auch im nächsten Jahrzehnt noch adäquat befriedigen zu können, wird unter der Bezeichnung LTE (Long Term Evolution) bereits die vierten Generation der Mobilfunkstandards entwickelt (4G). (Tanner, Hofstetter 2008). Durch diese Weiterentwicklung der UMTS-Technologie sollen die möglichen Übertragungsraten wesentlich erhöht werden. Um das Äußerste aus den verwendeten Frequenzbereichen herausholen zu können, steht dann langfristig wieder ein Generationswechsel bevor, der die Verwendung der auch bei WiMAX eingesetzten leistungsfähigeren und stabileren OFDM-Vielträger-Übertragung (Orthogonal Frequency Division Multiplex) statt der bei UMTS heute verwendeten WCDMA-Spreizbandtechnik (Wideband Code-Division Multiple Access) vorsieht.
Um zukünftig noch höhere Datenraten bei steigendem Verkehrsvolumen erreichen zu können, ist die Erschließung neuer Frequenzbänder in höherfrequenten Bereichen für den mobilen Datenfunk notwendig. So wird derzeit auch die Verwendung ehemaliger Frequenzen des analogen Fernsehens für den Mobilfunk diskutiert.

Zunehmende Probleme mit den quasioptischen Ausbreitungseigenschaften der elektromagnetischen Wellen in diesen Frequenzbereichen, insbesondere bei wechselnden Abschattungen und Mehrwegeempfang im mobilen Betrieb, erfordern dafür die Entwicklung immer komplexerer Übertragungs-, Modulationsund Fehlerkorrekturverfahren. Dabei wird die Entwicklung zukünftiger Mobilfunkstandards bisherige leitungsvermittelte Techniken ablösen und durch IP-basierte (Internet Protocol), paketvermittelte Netztechnologien ersetzen. Durch diese Entwicklung werden weitere Kapazitätserhöhungen und Kosteneinsparungen sowie neue, für den Kunden interessante Anwendungen erwartet.

Neben dem Mobilfunk drängen derzeit neue Funktechnologien auf den Markt (wie z. B. Bluetooth, Technologien in Verkehrssystemen, Diebstahlsicherungs-, Waren- und Personenerkennungssysteme und digitales Fernsehen). ${ }^{1}$

Bei breitbandigen Funkzugängen wird die Entwicklung in Richtung von noch mehr technischer Vielfalt gehen. Zur besseren Versorgung bisher mit Breitbanddiensten unterversorgter Gebiete werden zunehmend Funkanwendungen - zunächst WiMAX - eingesetzt werden. Möglicherweise gewinnen auch satellitengestützte Funkdienste an Bedeutung; letztere sind insbesondere für die Verteilung von MultimediaInhalten interessant (z. B. von mobilem Fernsehen wie DVB-H oder „Digital Video Broadcast“ an Handys oder Handhelds). Wegen der technisch problematischen Rückübertragung von Signalen von mobilen Geräten an Satelliten eignen sich diese Dienste weniger für Kommunikationsanwendungen, die auf bidirektionale Datenübertragung angewiesen sind.

\section{Wie sieht die Exposition der Bevölkerung mit EMF aus?}

Auskünfte über die Emissionen von Funksendeanlagen erteilt die EMF-Datenbank (Elektromagnetisches Feld) der Bundesnetzagentur für 
Elektrizität, Gas, Telekommunikation, Post und Eisenbahnen z. B. über ihre Internetseiten (http://www.bundesnetzagentur.de). Für den jeweiligen Standort einer Funksendestation können dort die exakten Immissionsdaten, $d . h$. die Strahlungswerte, die auf Mensch und Umwelt einwirken, abgefragt werden. Die konkreten Messdaten sollen darüber aufklären, welche Immissionen in der unmittelbaren Nähe von Sendeanlagen in Wohnbereichen und an Orten, die wie Schulen oder Kindergärten besonders im Fokus der öffentlichen Meinung stehen, tatsächlich auftreten.

Die Ergebnisse von zahlreich durchgeführten Messungen sind in 99 Prozent der Fälle folgende: Selbst bei höchster Leistung betragen die Immissionen weniger als ein Hunderttausendstel des Grenzwertes. Das verblüfft meist im hohen Maße die Mobilfunkmastgegner. Je nach Eigenschaft des Messpunktes (Entfernung zum Standort, Sichtverbindung etc.), schwanken die Immissionen typisch um bis zu drei Größenordnungen. Die höchsten Immissionen treten bei hoch montierten Antennen nicht unmittelbar neben der Antenne, sondern in 100 bis 300 Metern Entfernung auf.

Die neuen breitbandigen Dienste (z. B. WiMAX) werden im Vergleich $\mathrm{zu}$ anderen Hochfrequenzimmissionsquellen (Mobilfunk, Tonrundfunk, TV) diese bereits sehr niedrigen Werte nochmals um ein Vielfaches unterschreiten. Die GSM-Immissionen durch MobilfunkMasten liegen dagegen viel höher: Sie fallen im Vergleich etwa zehn- bis tausendfach höher aus. Die Sender für Mobilfunk müssen stärker strahlen, weil die Nutzer mobil sind und daher größere Reserven bei der Empfangsfeldstärke notwendig sind, während sich die Sender für den schnellen Internetzugang an stationäre Nutzer mit Außenantenne richten. Zudem handelt es sich bei WLAN und WiMAX um modernere Verfahren als GSM.

\section{Gesundheitspolitische Aspekte und Festlegung von Grenzwerten}

Besonders mit dem Ausbau der Mobilfunknetze (2. Generation, GSM, D- und E-Netze, 900 $\mathrm{MHz}, 1800 \mathrm{MHz}$ bzw. $1900 \mathrm{MHz}$ ) und dem Beginn der drahtlosen Massenkommunikation Anfang der 1990er Jahre sind diese Felder in die öffentliche Diskussion gekommen; sah man doch überall Sendemasten wie Pilze aus dem Boden sprießen. Die rasante Entwicklung der Mobilkommunikation mit immer mehr Sendern, immer mehr Empfängern, immer mehr und höheren Frequenzen zeigte Effekte. Der Begriff „Elektrosmog“ wurde geboren, wobei das bereits negativ belegte Wort „Smog“ verwendet wurde, um Sorgen und in manchen Fällen auch Ängsten vor negativen Folgen dieser Technik Ausdruck zu verleihen. Der Begriff „Elektrosmog“ erscheint unglücklich gewählt, da er in populistischer Manier Furcht vor einer Gefahr schürt, bevor deren Realität nachgewiesen ist.

Was wir brauchen, ist eine verantwortungsvolle Grundstrategie im Umgang mit den Risiken in der Industriegesellschaft. Entgleisungen der Technik, Missbrauch, oder auch ihre rücksichtslose Ausnutzung gegen Mensch und Natur müssen so weit wie irgend möglich vermieden werden. Das Streben nach Sicherheit, der Schutz von Mensch und Umwelt vor Gefahren aus der Techniknutzung sowie vor ungewolltem menschlichem Fehlverhalten ist dabei oberstes Gebot. Gleichzeitig gilt es aber, entwicklungsoffen und damit zukunftsfähig zu bleiben, die Technologien Schritt für Schritt weiter zu entwickeln, und sie so einzusetzen und zu nutzen, dass sie den angestrebten Zielen dienen, während man sich ihrer Risiken und der Verantwortung für die Gesellschaft bewusst bleibt.

Nationale und internationale Expertengremien nahmen sich diesem Problem an, und auch der Staat sah Handlungsbedarf. Zu nennen ist die 1992 gegründete ICNIRP (International Commission on Non-Ionizing Radiation Protection), deren Empfehlungen in Deutschland in die 26. Verordnung zur Durchführung des Bundes-Immissionsschutzgesetzes vom 26. Dezember 1996 (26 BImSchV) eingeflossen sind. $^{2}$ Es wurden Grenzwerte für die Stärke elektromagnetischer Felder festgelegt, die mit hohem Abstand $\mathrm{zu}$ nachweislich thermischen Effekten der Felder den Bereich der Unbedenklichkeit markieren.

Gleichzeitig lief die Forschung weiter auf Hochtouren und die Notwendigkeit, diese international zu koordinieren und deren Ergebnisse $\mathrm{zu}$ verfolgen und kontinuierlich auszuwerten, wurde dringendes Gebot. In Deutschland wurde die ,Forschungsgemeinschaft Funk“ (FGF) gegründet - eine von der Bundes- 
regierung, den Rundfunkanstalten und den Mobilfunkbetreibern finanzierte, jedoch unabhängige wissenschaftliche Gemeinschaft, der sich mehr als 50 Universitäten und Forschungsinstitute im In- und Ausland angeschlossen haben, und die unter Hinzuziehung internationaler Experten koordinierende wissenschaftliche Symposien veranstaltet sowie Forschungsprojekte vergibt und finanziert.

Eine wichtige Aktivität der FGF war die Einrichtung einer öffentlich zugänglichen Datenbank, die mit einem hohen Grad an Vollständigkeit wissenschaftliche, d. h. begutachtete (peer-reviewed) Studien zur Bewertung der Wirkung elektromagnetischer Felder (EMF) auf Mensch und Umwelt sammelt und nach einem übersichtlichen Schema auswertet. Diese Datenbank mit dem Namen ,EMF-Portal“ wird in Verantwortung der RWTH Aachen geführt und ist in den letzten Jahren auf einen Bestand von 12.500 Artikeln angewachsen (http://www.emfportal.de). Die Anzahl der Zugriffe auf diese Informationen aus dem In- und Ausland liegt inzwischen bei 1.500 bis 8.000 pro Tag.

\section{Zum Stand der Forschung}

Weltweit erscheinen wöchentlich neue wissenschaftliche Publikationen zu möglichen gesundheitlichen Wirkungen der Felder des Mobilfunks. In der überwiegenden Zahl der Untersuchungen sind die Ergebnisse negativ. Hin und wieder gibt es auch alarmierende Befunde; alarmierend sind sie allerdings mehr für die Presse als für die Wissenschaft, denn bei genauem Hinsehen sind diese „Positiv-Befunde“ bezüglich Applikationstechnik, Dosimetrie, Analysenmethodik oder Statistik oft sehr angreifbar. Und doch werden sie ernst genommen, was auch im Interesse der Sicherheit der Fall sein muss. Mitunter erfordern sie langwierige und teure Replikations-Studien, die in der Regel negativ ausgehen. Hierfür ließen sich mehrere Beispiele anführen. Besonders spektakulär war in letzter Zeit der Nachweis einer offensichtlichen Fälschung. So wurde in einem Wiener Labor angeblich nachgewiesen, dass selbst im Bereich zulässiger Intensitäten eines $1,8-\mathrm{GHz}-$ Feldes $(\mathrm{SAR}=2 \mathrm{~W} / \mathrm{kg})$ Einzel- und Doppelstrangbrüche der DNA zu beobachten seien (Diem et al. 2005). Frühere Negativ-Befunde dieser Art wurden von den Autoren nicht beachtet. Das Ergebnis war alarmierend. Sofort liefen weltweit Experimente an, diese Behauptung zu überprüfen, ohne jedoch ähnlich Effekte zu finden (Speit et al. 2007; Juutilainen et al. 2007; Valbonesi et al. 2008; Manti et al. 2008). Eine gründliche Analyse der Publikation, die mit einem Besuch in dem entsprechenden Labor verbunden war, konnte zumindest grobe methodische Fehler nachweisen, die als ,Letter to the Editor" publiziert wurden (Vijayalaxmi et al 2006; Ivancsits et al. 2005) Ungeachtet dessen erschien 2008 eine erneute Publikation, in welcher die umstrittenen Autoren ihre früheren Befunde bestätigten (Schwarz et al. 2008). Erst jetzt konnte nachgewiesen werden, dass die früheren und die jetzigen Experimente offenbar auf Fälschungen beruhen (Lerchl 2008).

Dieser Aufgabe haben sich inzwischen eine Reihe nationaler und internationaler Forschungsvorhaben angenommen. $\mathrm{Zu}$ nennen ist hier u. a. das im Jahr 2008 abgeschlossene Deutsche Mobilfunk-Forschungsprogramm, das in den letzten fünf Jahren 54 Forschungsprojekte auch ausländischer Institutionen mit insgesamt 17 Mio. Euro finanzierte. Dieses Forschungsprogramm enthält Untersuchungen auf allen Ebenen; sie reichen von molekularbiologisch-zytologischen Messungen über Tierversuche und Messungen an Probanden bis hin zu epidemiologischen Erhebungen.

Fasst man die Ergebnisse dieser und weiterer Untersuchungen zusammen, so ergibt sich, dass nur dann deutliche Effekte zu verzeichnen sind, wenn die Intensität der Exposition über den Grenzwerten liegt und zu einer Temperaturerhöhung im System führt. Es fehlt der reproduzierbare Nachweis für immer wieder behauptete „nicht-thermische“ Effekte unterhalb der Schwelle messbarer Temperaturerhöhungen in biologischen Geweben.

Eine Besonderheit scheinen die von mehreren Arbeitsgruppen gefundenen geringfügigen Änderungen im Elektroenzephalogramm (EEG) von Probanden zu sein, die mit ,gepulsten" Mobilfunkfeldern unterhalb oder in der Nähe des Grenzwertes exponiert waren. Obgleich auch die Autoren dieser Studien nicht von gesundheitsschädlichen Effekten sprechen - schließlich verursachen viele harmlose Umweltreize EEG-Effekte - so nimmt man diese Resultate doch ernst und bemüht sich um Re- 
produktion und Aufklärung des zugrunde liegenden Mechanismus. ${ }^{3}$ Möglicherweise ist eine Anregung von Thermorezeptoren dafür verantwortlich, die mit höchster Genauigkeit die Temperaturkonstanz im Gehirn regulieren.

In diesem Zusammenhang sind Messungen zu erwähnen die ergeben haben, dass bereits ein vom Betriebsstrom warmes Handy zu einer Erwärmung der Wange führen kann. Selbst die Wärme bzw. feuchtigkeitsisolierende Wirkung eines solchen Gerätes ist effektiver als der Diathermieeffekt der ausgesandten Felder, wenngleich diese in der Lage sind, zentimetertief in das Gewebe einzudringen.

\section{Neue Ansätze der Bewertung und weiterführende Empfehlungen}

Trotz der offensichtlich eindeutigen Ergebnisse der oben erwähnten Studien sind die Bedenken in Teilen der Bevölkerung noch nicht vollständig ausgeräumt. Verschiedene Umweltgruppen verweisen immer wieder auf Publikationen, die über Experimente mit positiven Effekten berichten. Wie ist damit umzugehen?

In regelmäßigen Abständen erscheinen Review-Artikel und Meta-Analysen. Es werden Versuche gemacht, die Ergebnisse der Forschung zusammenzufassen und zu Schlüssen zu kommen. Diese Meta-Analysen enthalten Tabellen der Publikationen mit den Vermerken $\mathrm{zu}$ verwendeten Parametern und erzielten Resultaten. Da in diesen Listen selbstverständlich alle Publikationen $\mathrm{zu}$ dem entsprechenden Thema verzeichnet sind, also auch jene, die durch Replikationen nicht verifiziert werden konnten, so lauten die Schlussfolgerungen in der Regel: „mixed results“, „mostly negative“, „no effects, but some with effects“, ,überwiegend keine Effekte“. Im letzten Satz heißt es dann zumeist: „Weitere Forschung ist erforderlich!“ Aussagen dieser Art lassen den Leser oft mehr verwirrt als informiert zurück.

Es stellt sich schnell die Frage: Wie viel Forschungsmittel sind noch einzusetzen, um zu einer endgültigen Einschätzung über eine mögliche Gefahr durch den Mobilfunk zu kommen? Genau genommen könnte schon heute ein Schlussstrich gezogen werden, wenn man nicht ohne Qualitätsstandards Mehrheiten suchen will oder sogar nur isolierte Forschungsergebnisse auswählt, die zum eigenen Weltbild pas- sen. Natürlich sollte man „wertend“ vorgehen, aber nach Maßstäben, die an wissenschaftlicher Qualität ausgerichtet sind.

Wenn auch das Resultat eines einzigen Experimentes ein ganzes System der Wissenschaft stürzen lassen kann, so kommt es doch darauf an, dass dieses Resultat stimmt, d. h. unter genau kontrollierten Bedingungen beliebig oft reproduzierbar ist. Außer den im Allgemeinen aufwändigen Reproduktionsexperimenten gibt es auch noch formale Maßstäbe, um die Qualität einer Publikation zu beurteilen. Dazu gehören nicht nur die oben erwähnten korrekten methodischen, d. h. „handwerklichen“ Qualitäten einer Arbeit, sondern auch Ansätze und Art der Auswertung eines Experiments. Trifft man z. B. auf Publikationen, die in den Abschnitten „Einleitung“ und „Diskussion“ nur solche Publikationen zitieren, die den Autoren genehm sind und widersprüchliche einfach ausblenden, dann disqualifiziert sich die Arbeit selbst.

Wie sollte es also weitergehen? Zunächst müsste man die Publikationen zu den einzelnen Gebieten (molekulargenetische, zellphysiologische, tierexperimentelle Studien, ProbandenExperimente, epidemiologische Erhebungen etc.) nicht zeitlich parallel, sondern chronologisch auswerten. Dies sollte in folgender Weise geschehen: „X hat einen wichtigen Effekt gefunden. Dieser wurde ernst genommen und von A, B, C etc. wiederholt. Der Effekt ließ sich nicht reproduzieren. Offensichtlich sind die Ergebnisse von $\mathrm{X}$ falsch und können gestrichen werden!" Eine solche Analyse würde Luft schaffen und zumindest denen den Wind aus den Segeln nehmen, die immer wieder alte, längst wissenschaftlich abgelegte Resultate argumentativ wiederholen. Andererseits würde sich auch zeigen, dass einige der „positiven“ Effekte noch nicht kontrolliert wurden. Wenn man sie nicht von vornherein als methodisch fehlerhaft ausschließen kann, oder wenn sie sich nicht dadurch falsifizieren lassen, dass die notwendigerweise dabei auftretenden Folgeerscheinungen nicht nachweisbar sind, dann würde es sich dringend empfehlen, diese Experimente zu wiederholen (natürlich von einem anderen Labor, möglichst aber in Kooperation mit jenem, das diesen Effekt glaubt gefunden zu haben).

Eine zweite Forderung wäre die verstärkte Anwendung sogenannter Positiv-Kontrollen. In guten Arbeiten ist dies längst zur Regel gewor- 
den. Fragt man z. B. nach möglichen genetischen Effekten hochfrequenter Felder, so setzt man in Parallelexperimenten Gamma-Strahlung ein, von der solche Effekte bekannt sind und testet damit die Empfindlichkeit des Systems. Häufig fehlen solche Kontrollen jedoch bezüglich der Entscheidung: ,thermisch nicht-thermisch“. Zumeist wird ein Effekt „nicht-thermisch“ genannt, weil eine Temperaturerhöhung im Organismus oder im Medium nicht nachweisbar ist. Man ignoriert dabei die Reaktion lokaler Thermorezeptoren, die bekannterweise außerordentlich empfindlich sind, und die eventuell durch Absorption der HFFelder selektiv angeregt werden. Warum vergleicht man nicht z. B. die durch GSM-Felder verursachten EEG-Effekte mit gepulsten Infrarot-Strahlen? Inzwischen gibt es genaue Untersuchungen zur Expression sogenannter Hitzeschock-Proteine (HSP) bei gezielter konventioneller Erwärmung (Cranfield et al. 2004; Prahlad et al. 2008; Franzmann et al. 2008).

Die gleichen Experimente müssten gezielt wiederholt werden, indem die Systeme durch Hochfrequenzfelder diathermisch erwärmt werden. Solche Experimente könnten übrigens auch in der Therapie gewinnbringend sein. Es ist bisher nicht untersucht, ob sich diese Therapie nicht vielleicht optimieren ließe, wenn man nicht kontinuierliche, sondern gepulste Felder verwenden würde.

Entscheidend bei der Bewertung gefundener Effekte sollte letztlich deren Gesundheitsrelevanz sein. Zumeist löst ein solches Resultat in der Presse zunächst einen Aufschrei aus über angeblich nachgewiesene Gefahren des Mobilfunks. Selten wird gefragt: Ist das ein „AlltagsEffekt"? Wie verhält sich diese Abweichung in Relation zu täglichen Beeinflussungen durch Wärmequellen, Höhenstrahlungen oder natürlicher Radioaktivität? Aus Gründen des Gesundheitsschutzes könnte diese Art von Effekten vernachlässigt werden. Die Wissenschaft sollte sich jedoch sehr wohl weiter dafür interessieren, könnte sich dahinter vielleicht ein bislang unbekannter Mechanismus verbergen.

Der Mechanismus der Wirkung hochfrequenter elektromagnetischer Felder ist ein Forschungsgebiet, das so alt ist wie die technische Erzeugung dieser Felder selbst. Man ist heute nicht nur in der Lage, aus Kenntnis der elektrischen Parameter des biologischen Gewebes die dort absorbierte Energie bzw. Leistung zu errechnen (SAR-Wert in W/kg - Specific Absorption Rate), und daraus die zu erwartende Erwärmung zu ermitteln; man kennt auch direkte Einflüsse der elektrischen Komponente dieser Felder auf Ladungen und Dipole. Dies wird in der Biophysik mit Recht als ,nichtthermisch" bezeichnet, auch wenn es dabei zu einer Erwärmung kommt, denn thermodynamisch muss sich letztlich jede absorbierte Energie in Wärme umwandeln. Solche Effekte sind unter der Bezeichnung „Dielektrophorese“ und „Elektrorotation" theoretisch und experimentell hinlänglich gesichert, treten jedoch erst bei Feldstärken auf, die einige Zehnerpotenzen über denen liegen, die für den Umweltschutz relevant sind. Somit haben sie lediglich ihre Bedeutung in der Biotechnologie.

Trotz jahrzehntelanger Forschung und zahlreicher Ansätze ist es bisher nicht gelungen, Mechanismen $\mathrm{zu}$ finden, nach denen hochfrequente elektromagnetische Felder unterhalb der thermischen Schwelle der Thermorezeptoren wirksam sein könnten. Da solche „nicht-thermischen“ Effekte im herkömmlichen Gebrauch des Wortes bisher auch noch nicht zweifelsfrei nachgewiesen werden konnten, scheint Übereinkunft zwischen Theorie und Experiment $\mathrm{zu}$ bestehen. Im Bereich thermischer Wirkungen gibt es inzwischen viele Modelle unter Berücksichtung der dielektrischen Heterogenität des Gewebes und dessen Thermoregulation über die Durchblutung. Hier ist ein stetiger Fortschritt zu verzeichnen, der jedoch sowohl bezüglich mikrothermischer Effekte als auch der Berücksichtigung molekularer Thermorezeptoren noch Lücken aufweist.

Die Bedeutung der Forschung zu Wirkungsmechanismen liegt auf der Hand. Nur auf dieser Basis kann der Begriff der „Dosis“ definiert werden. Der heute gebräuchliche SARWert berücksichtigt die thermischen Effekte ohne Beachtung einer eventuellen Akkumulation, die sich im Gegensatz zur Wirkung ionisierender Strahlung im Bereich der Frequenzen des Mobilfunks allerdings bisher auch noch nicht nachweisen ließ. Nur durch genaue Kenntnis der Mechanismen ist man ferner in der Lage, die Abhängigkeit des Effektes von verschiedenen Parametern (z. B. Frequenz, Pulsation, Intensität) vorauszusagen und die Wirkung auf andere 
Bedingungen, $d . h$. auch auf Immissionen neuer, zukünftiger Technologien zu extrapolieren.

\section{Schlussbemerkung}

Zusammenfassend lässt sich sagen: Gesundheitsbezogene Richtlinien zum Schutz der Gesundheit vor elektromagnetischen Feldern (EMF) erfordern die Bewertung der gesundheitlich nachteiligen Wirkungen einer Exposition durch EMF auf der Basis des fundierten wissenschaftlichen und medizinischen Wissens.

Eine solche Bewertung muss frei von wirtschaftlichen und politischen Interessen sein. Die internationale Kommission zum Schutz vor nicht-ionisierender Strahlung (ICNIRP), in der als wissenschaftlich unabhängiger Organisation alle relevanten Disziplinen vertreten sind, ist dafür qualifiziert, die Aufgabe der Risikobewertung gemeinsam mit der Weltgesundheitsorganisation durchzuführen. ${ }^{4}$

Meine persönliche Einschätzung für die Zukunft ist, dass sich auch mit zunehmendem Einsatz der im Artikel beschriebenen neuen breitbandigen „Funkanwendungen“ die Expositionslage der Bevölkerung nicht wesentlich verändern wird und dass die bestehenden Vorsorgemaßnahmen zum Schutz der Bevölkerung auch auf absehbare Zeit ausreichen werden.

\section{Anmerkungen}

1) Einschläge Ausführungen zu diesen Weiterentwicklungen und neuen Techniken finden sich bei Dahlman et al. (2008); Aravantinos, Fallah (2008) und 3G Americas (2007); siehe dazu auch die „Internationale Standardisierung im Bereich drahtloser Kommunikation: International Telecommunication Union - Radiocommunication Sector" (ITU-R) (http://www.itu.int/ITU-R/) und Standardisierungsgruppe „Third Generation Partnership Project" (3GPP) (http://www.3gpp.org/).

2) Die 26. Verordnung zur Durchführung des Bundes-Immissionsschutzgesetzes vom 26. Dezember 1996 findet sich unter http://www.gesetze-iminternet.de/bundesrecht/bimschv_26/gesamt.pdf (download 13.12.08).

3) Siehe dazu insbesondere Regel et al. (2007) und als kritischen Widerpart Balzano, Swicord (2008).

4) ICNIRP ist die - formal von der Weltgesundheitsorganisation, der ILO (International Labour Organisation) und der Europäischen Union - anerkannte regierungsunabhängige Organisation für den Schutz der Gesundheit vor einer Exposition mit nicht-ionisierender Strahlung. Die Abstimmung ihrer Empfehlungen erfolgt mit den vier ständigen Ausschüssen und den beratenden Experten der Kommission, mit den der IRPA (International Radiation Protection Association) angeschlossenen nationalen Verbänden und über zusätzliche Experten.

\section{Literatur}

$3 G$ Americas, 2007: Defining 4G: Understanding the ITU Process for the Next Generation of Wireless Technology. June 2007; http://3gameri cas.com/PDFs/3G_Americas_Defining_4G_WP_Jul y2007.pdf (download 13.12.08)

Aravantinos, E.; Fallah, M.H., 2008: Potential Scenarios and Drivers of the 4G Evolution. ITS 17th Biennial Conference, Montreal, June 24-27; http:// www.canavents.com/its2008/abstracts/169.pdf (download 13.12.08)

Balzano, Q.; Swicord, M., 2008: Comments on Neurophysiological Effects of Mobile Phone Electromagnetic Fields on Humans: a Comprehensive Review. In: Bioelectromagnetics 29 (2008), S. 410

Cranfield, C.G.; Dawe, A.; Karloukovski, V. et al., 2004: Biogenic Magnetite in the Nematode Caenorhabditis Elegans. In: Proc. Royal Soc. London, Series B Biological Sciences 271 (2004), S. 436-439

Dahlman, E.; Parkvall, S.; Sköld, J. et al., 2008: 3G Evolution - HSPA and LTE for Mobile Broadband. Oxford (2. Ausgabe)

Diem, E.; Schwarz, C.; Adlkofer, F. et al., 2005: Non-thermal DNA Breakage by Mobile-phone Radiation $(1800 \mathrm{MHz})$ in Human Fibroblasts and in Transformed GFSH-R17 Rat Granulosa Cells In Vitro. In: Mutat. Res. 583/2 (2005): S. 178-183

Franzmann, T.M.; Menhorn, P.; Walter, S. et al., 2008: Activation of the Chaperone Hsp26 is Controlled by the Rearrangement of its Thermosensor Domain. In: Molecular Cell 29 (2008), S. 207-216

Ivancsits, S.; Pilger, A.; Diem, E. et al., 2005: Cell Type-specific Genotoxic Effects of Intermittent Extremely Low-frequency Electromagnetic Fields. In: Mutat. Res. 583 (2005), S. 184-188

Juutilainen, J.; Heikkinen, P.; Soikkeli, H. et al., 2007: Micronucleus Frequency in Erythrocytes of Mice after Long-term Exposure to Radiofrequency Radiation. In: Int. J. Radiat. Biol. 83 (2007), S. 213-220

Lerchl, A., 2008: Fälscher im Labor und ihre Helfer: Die Wiener Mobilfunk-Studien - Einzelfall oder Symptom? Norderstedt

Manti, L.; Braselmann, H.; Calabrese, M.L. et al. 2008: Effects of Modulated Microwave Radiation at 
Cellular Telephone Frequency $(1.95 \mathrm{GHz})$ on XRay-induced Chromosome Aberrations in Human Lymphocytes In Vitro. In: Radiat. Res. 169 (2008), S. $575-583$

Prahlad V, Cornelius T, Morimoto R.I., 2008: Regulation of the Cellular Heat Shock Response in Caenorhabditis Elegans by Thermosensory Neurons. In: Science 320 (2008), S. 811-814

Regel, S.J.; Gottselig, J.M.; Schuderer, J. et al., 2007: Pulsed Radio Frequency Radiation Affects Cognitive Performance and the Waking EEG. In: Neuroreport 18 (2007), S. 803-807

Schwarz, C.; Kratochvil, E.; Pilger, A. et al., 2008: Radiofrequency Electromagnetic Fields (UMTS, $1,950 \mathrm{MHz}$ ) Induce Genotoxic Effects In Vitro in Human Fibroblasts but not in Lymphocytes. In: Int Arch Occup Environ Health 81 (2008), S. 755-767

Speit, G.; Schuetz, P.; Hoffmann, H., 2007: Genotoxic Effects of Exposure to Radiofrequency Electromagnetic Fields (Rf-Emf) in Cultured Mammalian Cells Are Not Independently Reproducible. In: Mutat. Res. 626 (2007), S. 42-47

Tanner, R.; Hofstetter, R., 2008: LTE, der nächste Mobilfunkstandard. In: Bulletin SEV/VSE 15 (2008), S. 9-12, http:/www.fh-htwchur.ch/uploads/ media/TannerHofstter_ArtikelAug08.pdf

Valbonesi, P.; Franzellitti, S.; Piano, A. et al., 2008: Evaluation of HSP70 Expression and DNA Damage in Cells of a Human Trophoblast Cell Line Exposed to $1.8 \mathrm{GHz}$ Amplitude-modulated Radiofrequency Fields. In: Radiat. Res. 169 (2008), S. 270-279

Vijayalaxmi; McNamee, J.; Scarfi, M.R., 2006: Comments on: "DNA Strand Breaks" by Diem et al. [Mutat. Res. 583 (2005), S. 178-183] and Ivancsits et al. [Mutat. Res. 583 (2005), S. 184-188]. Mutat. Res. 603/1 (2006), S.104-106

\section{Kontakt}

Dr. Gerd Friedrich

Forschungsgemeinschaft Funk e.V. (FGF)

Rathausgasse 11 A, 53111 Bonn

Tel.: +49 (0) 228 / 72622 - 0

E-Mail: friedrich@fgf.de

$\ll 》$

\section{Forschungsfelder und wissen- schaftliche Risikodiskussion} Zusammenfassende Darstellung und Einordnung der Ergebnisse des Deutschen MobilfunkForschungsprogramms

\section{von Wolfgang Weiss und Rüdiger Matthes, BfS Oberschleißheim, sowie Christoph Revermann, TAB Berlin}

Das Deutsche Mobilfunk-Forschungsprogramm (DMF) diente der Aufklärung gesundheitlicher Risiken des Mobilfunks. Mit einer Analyse, zu der auch ein multidisziplinärer Interpretationsansatz gehörte, wurde allen Hinweisen nachgegangen, die in der wissenschaftlichen Literatur anzutreffen waren. Beteiligt waren alle einschlägigen naturwissenschaftlich / technischen, biologisch / medizinischen sowie epidemiologischen Fachdisziplinen. Erstmals wurden im DMF auch Fragen der Wahrnehmung möglicher Risiken in der Bevölkerung, der Vermittlung einschlägiger Informationen an die Bedarfsträger sowie Möglichkeiten der Prävention und Schlichtung von Konflikten gezielt verfolgt. Die im DMF erarbeiteten wissenschaftlichen Erkenntnisse haben insgesamt die bestehenden Grenzwerte bestätigt. Für die zukünftige Forschung haben zwei Fragen Priorität: Welche Langzeitrisiken bei Handynutzungszeiten von mehr als zehn Jahren sind zu erwarten? Reagieren Kinder empfindlicher auf die Felder des Mobilfunks als Erwachsene? ${ }^{1}$

\section{Forschung und Standardsetzung für den gesundheitlichen Strahlenschutz}

Die Festlegung von Standards zum Schutze vor gesundheitlichen Gefahren und Risiken ionisierender und nicht-ionisierender Strahlung ist ein gesamtgesellschaftlicher Prozess, der auf der Bewertung aller vorliegenden wissenschaftlichen Erkenntnisse basiert. Im Bereich der elektromagnetischen Felder des Mobilfunks wurden in den letzten Jahrzehnten einige zehntausend einschlägige wissenschaftliche Originalpublikationen veröffentlicht. Diese werden von internationalen und nationalen Fachgremien wie der ICNIRP (International Commission on Non-Ionizing Radiation Protection), der WHO (World Health Organization) und der 